\title{
Active Radiative Thermal Switching with Graphene Plasmon Resonators
}

\author{
Ognjen llic, Nathan H Thomas, Thomas Christensen, Michelle C. Sherrott, \\ Marin Soljacic, Austin J. Minnich, Owen D Miller, and Harry A Atwater \\ ACS Nano, Just Accepted Manuscript • DOI: 10.1021/acsnano.7b08231 • Publication Date (Web): 12 Mar 2018 \\ Downloaded from http://pubs.acs.org on March 14, 2018
}

\section{Just Accepted}

"Just Accepted" manuscripts have been peer-reviewed and accepted for publication. They are posted online prior to technical editing, formatting for publication and author proofing. The American Chemical Society provides "Just Accepted" as a service to the research community to expedite the dissemination of scientific material as soon as possible after acceptance. "Just Accepted" manuscripts appear in full in PDF format accompanied by an HTML abstract. "Just Accepted" manuscripts have been fully peer reviewed, but should not be considered the official version of record. They are citable by the Digital Object Identifier (DOI®). "Just Accepted" is an optional service offered to authors. Therefore, the "Just Accepted" Web site may not include all articles that will be published in the journal. After a manuscript is technically edited and formatted, it will be removed from the "Just Accepted" Web site and published as an ASAP article. Note that technical editing may introduce minor changes to the manuscript text and/or graphics which could affect content, and all legal disclaimers and ethical guidelines that apply to the journal pertain. ACS cannot be held responsible for errors or consequences arising from the use of information contained in these "Just Accepted" manuscripts. 


\title{
Active Radiative Thermal Switching with Graphene Plasmon Resonators
}

\author{
Ognjen Ilic, ${ }^{1}$ Nathan H. Thomas, ${ }^{2}$ Thomas Christensen, ${ }^{3}$ Michelle C. Sherrott, ${ }^{1}$ Marin Soljačić, ${ }^{3}$ Austin J. \\ Minnich, ${ }^{2}$ Owen D. Miller, ${ }^{4}$ and Harry A. Atwater ${ }^{1}$ \\ ${ }^{I}$ Department of Applied Physics and Materials Science, California Institute of Technology, \\ Pasadena, CA 91125, USA \\ ${ }^{2}$ Division of Engineering and Applied Science, California Institute of Technology, Pasadena, CA \\ 91125, USA \\ ${ }^{3}$ Department of Physics, Massachusetts Institute of Technology, Cambridge, MA 02139, MA, USA \\ ${ }^{4}$ Department of Applied Physics and Energy Sciences Institute, Yale University, New Haven, CT \\ 06511, USA
}

\begin{abstract}
We theoretically demonstrate a near-field radiative thermal switch based on thermally excited surface plasmons in graphene resonators. The high tunability of graphene enables substantial modulation of near-field radiative heat transfer, which, when combined with the use of resonant structures, overcomes the intrinsically broadband nature of thermal radiation. In canonical geometries, we use nonlinear optimization to show that stacked graphene sheets offer improved heat conductance contrast between "ON" and "OFF" switching states, and that a $>10 \mathrm{x}$ higher modulation is achieved between isolated graphene resonators than for parallel graphene sheets. In all cases, we find that carrier mobility is a crucial parameter for the performance of a radiative thermal switch. Furthermore, we derive shape-agnostic analytical approximations for the resonant heat transfer that provide general scaling laws and allow for direct comparison between different resonator geometries dominated by a single mode. The presented scheme is relevant for active thermal management and energy harvesting as well as probing excited-state dynamics at the nanoscale.
\end{abstract}

Keywords: graphene, thermal radiation, near-field radiative heat transfer, surface plasmon

Radiative heat transfer on the nanoscale holds promise for next-generation energy conversion technologies, including heat-to-electricity conversion platforms such as near-field thermophotovoltaics and near-field solid-state refrigeration. A key enabler is the idea that closely separated objects at different temperatures-i.e. objects at separation distances much smaller than the characteristic thermal wavelength - can exhibit order-of-magnitude increases in the radiatively exchanged power relative to the power that can be transferred in the far field. While the early work on near-field radiative heat transfer (NF-RHT) focused on the thermal energy exchange between conducting plates, ${ }^{1,2}$ the advancements in nanofabrication have led to experimental demonstrations of NF-RHT in a number of configurations. ${ }^{3-17}$ Among recent studies, radiative nanoscale energy transfer has been investigated in metasurfaces, ${ }^{18}$ non- 
reciprocal systems and systems with gain, ${ }^{19,20}$ van der Waals stacks, ${ }^{21}$ and for concepts such as luminescent refrigeration, ${ }^{22}$ thermal extraction, ${ }^{23}$ thermal rectification and amplification, ${ }^{24-27}$ and radiative heat transfer limits. ${ }^{28-30}$

A key functionality central to the application of NF-RHT is a means of active heat transfer control - a scheme whereby external parameters can dynamically modulate the radiative flux between objects without necessitating a temperature change. The challenge of realizing such a thermal switch is two-fold: (1) the broadband spectrum of thermal radiation makes it difficult to modulate the radiative heat transfer to a significant degree, and (2) such a switch must comprise materials with tunable emissivity at a fixed temperature. Here, we propose use of coupled graphene resonators as a means to overcome both challenges; their highly tunable optical properties allow for constant-temperature operation, and provide a means to dramatically modulate NF-RHT despite the broadband nature of thermal radiation. In contrast to their bulk counterparts, low-dimensional plasmonic materials such as graphene exhibit highly tunable optical properties when electrically biased. Moreover, graphene supports strongly confined surface plasmons in the technologically important thermal IR spectral range. Finally, graphene combines a strong optical response with low losses, endowing it with the largest optical response of known plasmonic materials. ${ }^{30}$ Jointly, these attributes have sparked a significant interest in the study of plasmon-mediated NF-RHT in graphene. ${ }^{31-40}$

In this work, we find that optimal combinations of resonator size and material properties, specifically carrier concentration and relaxation rate, can enable large thermal switching ratios and high levels of modulation sensitivity. The working principle behind thermal switching with plasmonic graphene resonators is the ability to dynamically tune the modes of the resonances of the emitting and the absorbing objects into and out of resonance. We illustrate the idea of a thermal switch in several relevant configurations, including thermal switching between (a) graphene sheets, (b) multilayer graphene stacks, (c) dipolar graphene resonators, and (d) hybrid resonator-multilayer structures (Fig. 1). In this radiative heat transfer analysis with multiple configurations and inputs (e.g. temperature, distance, etc.), we identify carrier mobility as a critical parameter in achieving a large contrast between the "ON" (maximal heat transfer) and "OFF" (minimal heat transfer) states: higher mobility gives rise to sharper plasmonic resonances that are more easily detuned. For each value of mobility, identifying the ON and OFF thermal conductance states constitutes a nonlinear optimization problem over a parameter space of all allowable gate voltages. We find that in all structures, ON states comprise similarly doped graphene structureswhere the individual plasmonic resonances of the two sides efficiently overlap — and where optimal Fermi levels depend on carrier mobility and resonator size. For analyzed OFF states, we identify relevant regimes that depend on carrier mobility. Finally, we derive analytical approximations that highlight the relevant scaling laws and key parameters, and show that heat flux modulation is possible even with graphene on infrared active substrates.

\section{RESULTS AND DISCUSSION}

The radiative energy flux exchanged between two structures of temperatures $T_{1}$ and $T_{2}$ is given by ${ }^{41}$ 
where $\Theta(\omega, T)=\hbar \omega /\left[\exp \left(\hbar \omega / k_{\mathrm{B}} T\right)-1\right]$ is the mean energy of a photon, and $\Phi$ is the spectral transfer function which accounts for the geometry, shape, and (temperature-dependent) material properties of the two objects. In this work, we focus on the radiative thermal conductance (RTC) $h$ between two structures, defined for a given temperature $T$ as $h(T)=\lim _{T_{1}, T_{2} \rightarrow T} H\left(T_{1}, T_{2}\right) /\left(T_{1}-T_{2}\right)=\int_{0}^{\infty} \mathrm{d} \omega \frac{\partial \Theta}{\partial T}(\omega, T) \Phi(\omega, T)$. As a first step in our analysis, we examine the radiative heat transfer between two graphene sheets, as shown in Fig. 1a. For two parallel graphene sheets radiatively exchanging heat in the near field, and separated by a distance $d$, the spectral transfer function per unit area is ${ }^{33,34}$

$$
\Phi_{\text {sheets }}(\omega)=\frac{1}{\pi^{2}} \int_{\omega / c}^{\infty} \mathrm{d} q q \frac{\operatorname{Im}\left[r_{1}\right] \operatorname{Im}\left[r_{2}\right]}{\left|1-r_{1} r_{2} \mathrm{e}^{2 \mathrm{i} \kappa d}\right|^{2}} \mathrm{e}^{2 \mathrm{i} \kappa d},
$$

where $q$ and $\kappa$ are the in-plane and the perpendicular wave-vector, respectively $\left(\kappa=\sqrt{\omega^{2} / c^{2}-q^{2}}\right)$, and $r_{i}$ is the reflection coefficient of the $i$-th sheet (related to the, generally nonlocal, graphene surface conductivity, see SI). In this configuration, the radiative thermal conductance $h$ depends on several physical parameters: $h=h\left(E_{i}, \mu_{i}, T, d\right)$, where $E_{i}=\left(E_{1}, E_{2}\right)$ and $\mu_{i}=\left(\mu_{1}, \mu_{2}\right)$ denote the Fermi levels and carrier mobilities of the two sheets, respectively, $T$ is the temperature, and $d$ is the separation. Because both $E_{1}, E_{2}$ are actively tunable through electrostatic gating, our goal is to determine the optimal pairs $\left(E_{1}^{\text {on }}, E_{2}^{\text {on }}\right)$ and $\left(E_{1}^{\text {off }}, E_{2}^{\text {off }}\right)$ that correspond to the ON and OFF states, namely where $h_{\text {on }} \equiv$ $h\left(E_{1}^{\text {on }}, E_{2}^{\text {on }}\right)$ and $h_{\text {off }} \equiv h\left(E_{1}^{\text {off }}, E_{2}^{\text {off }}\right)$. A thermal switch with excellent modulation ability will then have a high switching ratio $\eta=h_{\text {on }} / h_{\text {off }}$.

Figure 2a shows the maximum conductance $h_{\text {on }}$ and the switching ratio $\eta$ as a function of the carrier mobility. We assume equal mobilities $\mu_{1,2}=\mu$, and fix the temperature $(T=300 \mathrm{~K})$ and the sheet separation $(d=100 \mathrm{~nm})$. Carrier mobility quantifies the magnitude of optical losses in graphene and is related to the carrier relaxation time $\tau$ via the impurity-limited approximation $\tau=\mu E_{\mathrm{F}} / e v_{\mathrm{F}}^{2} \cdot{ }^{42}$ For each value of mobility, we find the optimal $E_{1,2}^{\text {on }}$ and $E_{1,2}^{\text {off }}$ pairs in the allowable range $E_{i} \in\left[E_{\min }, E_{\max }\right]$. For the allowable range, we assume $E_{\min } \sim k_{\mathrm{B}} T$ and $E_{\max }=0.6 \mathrm{eV}$, consistent with typical experimental gate voltages (we note that presented results are not sensitive to the choice of $E_{\min }$, whether it is zero or $k_{\mathrm{B}} T$ ). The $E_{1,2}^{\text {on }}$ and $E_{1,2}^{\text {off }}$ pairs are computed numerically using a (multi-start) local, derivative-free, optimization algorithm. ${ }^{43-45}$ For the case of two graphene sheets radiatively exchanging heat in Fig. 2a, we observe a peak in the maximum conductance $h_{\text {on }}$ (solid black), implying the existence of an optimal optical loss rate which maximizes the heat transfer. The existence of optimal loss arises from the geometry of the problem. A parallel plate configuration, due to multiple reflections between the plates, does not achieve the optimal-absorber condition, exhibiting a heat transfer rate that is substantially weaker than the extended-structure limit. ${ }^{29}$ Because of this, we do not expect the optical response and the heat transfer rate to monotonically increase with mobility (or, equivalently, decrease with mounting optical loss). For the parameters under analysis here, we find the optimal mobility for the case of two graphene sheets to be $\mu_{\mathrm{opt}} \approx 1800 \mathrm{~cm}^{2} / \mathrm{Vs}$, and the corresponding radiative thermal conductance $h_{\mathrm{on}} / h_{\mathrm{bb}} \approx 340$ for $E_{1}^{\text {on }}=$ 
$E_{2}^{\text {on }}=0.173 \mathrm{eV}$. Here, the conductance is normalized to the far-field limit of radiatively coupled blackbodies (with unity view factor) $h_{\mathrm{bb}}(T)=\frac{\mathrm{d}}{\mathrm{d} T}\left(\sigma_{\mathrm{SB}} T^{4}\right)=4 \sigma_{\mathrm{SB}} T^{3}$, where $\sigma_{\mathrm{SB}}$ is the Stefan-Boltzmann constant. We note that in all cases, the emitter-receiver symmetry ensures that the ON state comprises equally doped graphene sheets $\left(E_{1}^{\text {on }}=E_{2}^{\text {on }}\right.$ ), such that the resonances are aligned (Fig. 2c). For analyzed OFF states, the carrier mobility is relevant. For low carrier mobility, maximal detuning of broad plasmonic resonances is achieved at the extremes of the allowable range of Fermi levels. In contrast, for higher carrier mobilities, once the plasmonic resonances are sufficiently detuned, the heat flux suppression in the OFF state is reduced with further separation of the emitter/absorber Fermi levels due to the onset of the interband transition in the lower-doped graphene structure (and the corresponding additional contribution to the radiative heat transfer).

In contrast to the heat transfer rate, the switching ratio $\eta$ monotonically increases with carrier mobility (solid, red in Fig. 2a), which reduces the plasmonic linewidths and thus enables improved detuning of resonances. We also observe (Fig. 2c) a cross-over value of mobility $\left(\sim 1300 \mathrm{~cm}^{2} / \mathrm{Vs}\right)$ that separates the two regimes of $\eta$ : for low mobility (i.e. broad resonances), the OFF state is achieved for the end values of the range of allowable Fermi levels, namely $E_{1(2)}^{\text {off }}=E_{\min (\max )}$; in contrast, for higher mobility $E_{1}^{\text {off }}>E_{\min }$ and the switching ratio increases faster with increasing mobility. Despite the multiple reflections in the parallel-plate geometry and the failure to achieve the optimal-absorber condition, the switching ratio can be appreciable, reaching a value of $\eta \approx 8.5$ for $\mu=10^{3} \mathrm{~cm}^{2} / \mathrm{Vs}$ (and $\eta \approx 45$ for $\mu=10^{4} \mathrm{~cm}^{2} / \mathrm{Vs}$ ).

The concept of thermal switching using two graphene sheets can be further extended to parallel graphene stacks (Fig. 1b). As an example, we focus on the near-field radiative heat transfer between a single graphene sheet (object 1) and a stack comprising two graphene sheets in close proximity (object 2). We fix the separation between the sheets in the second stack at $\delta=10 \mathrm{~nm}$ and object separation, as before, at $d=100 \mathrm{~nm}$. In this case, active modulation is achieved with parameters $E_{i}=\left(E_{1}, E_{21}, E_{22}\right)$, as sketched in Fig. 2a. Similar to the 2-sheet case, we also observe the existence of an optimal mobility that maximizes the radiative thermal conductance (blue, dashed, in Fig. 2a). In addition, we note a slight decrease $(20 \%)$ of $h_{\text {on }}$ relative to the 2 -sheet case, which can be attributed to the inability to achieve perfectly resonant coupling in this asymmetric configuration. The condition for maximal RTC is reached for a nearly-symmetric configuration $E_{1} \sim E_{21}$ and $E_{22} \sim E_{\min }$. Despite the optimal low carrier concentration of the bottom sheet, its optical response is appreciable enough to detune the plasmonic resonance, resulting in a decrease of $h_{\text {on }}$.

While the presence of the bottom sheet in the stack reduces the maximum heat transfer rate, it in turn enables a noticeably larger switching ratio. The improved switching ratio arises from the $h_{\text {off }}$ suppression due to resonance blue-shift in the graphene stack. This effect is elucidated by examining the local density of states (LDOS) above a layered stack. The LDOS at a point $\boldsymbol{r}$ is proportional to the decay rate of an (orientation-averaged) dipole at that point, given by ${ }^{46} \rho(\boldsymbol{r}, \omega)=\left(2 \omega / \pi c^{2}\right) \operatorname{Im}\{\operatorname{Tr}[\overleftrightarrow{\boldsymbol{G}}(\boldsymbol{r}, \boldsymbol{r}, \omega)]\}$, where $\overleftrightarrow{\boldsymbol{G}}$ is the dyadic Green function (SI). Fig. 3a shows the spectral LDOS above a graphene stack of $N$ identically doped sheets (of mutual, constant sheet-separation $\delta$ ). For $N>1$ the one-sheet plasmon 
dispersion fractures into a set of $N$ hybridized resonances, split into mutually bonding and anti-bonding modes, corresponding to low- and high-frequency branches. The principal LDOS contribution originates from the highest frequency branch. Normalizing the stack's LDOS to that of an individual sheet, we observe a substantially enhanced optical response at higher frequencies (Fig. 3b). Finally, Fig. 3c shows the $(k, \omega)$ dependence of the LDOS and the relevant higher order modes of the graphene stack. Together, these considerations further elucidate the previously noted blue shift of the two-sheet case with $E_{21,22}^{\text {off }}=$ $E_{\max }$ relative to the single sheet case with $E_{2}^{\text {off }}=E_{\max }$. In summary, while the maximum thermal conductance $h_{\text {on }}$ suffers from the introduction of the bottom sheet, the reduction is more than made up by the lower $h_{\text {off }}$, thus leading to an enhanced switching ratio $\eta$.

Moving beyond extended structures, we analyze radiative heat transfer between isolated graphene resonators, as shown in Fig. 1c. In the dipolar limit, the spectral transfer function for resonators 1 and 2 (normalized to resonator area $A$ ) can be expressed as $\Phi(\omega, d)=\frac{1}{8 \pi^{3}} \frac{1}{d^{6}} \sum_{j \in \hat{r}} \lambda_{j} \operatorname{Im}\left[\alpha_{1}^{j}\right] \operatorname{Im}\left[\alpha_{2}^{j}\right] / A$ where $d$ is the resonator separation distance, $\alpha_{1(2)}$ is the polarizability of resonator $1(2)$, and $\lambda_{j}$ is a numerical prefactor that depends on the relative orientation of the two resonators (SI). The polarizability connects the induced dipole moment $p(\omega)=\epsilon_{0} \alpha(\omega) E_{0}$ with an external field $E_{0}$, and can be expressed as the eigenmode sum $^{47}$

$$
\alpha(\omega)=2 L^{3} \sum_{v} \frac{\Delta_{v}}{\zeta_{v}-\zeta(\omega)},
$$

where the geometrical shape of a graphene resonator is captured by the normalized eigenfrequencies $\zeta_{v}$ and the oscillator strengths $\Delta_{v}$. The size and the material-response dependence of the graphene resonator are embedded in the dispersive parameter $\zeta(\omega)=2 \mathrm{i} \epsilon_{0} \epsilon \omega L / \sigma(\omega)$, where $L$ is the characteristic length scale and $\sigma(\omega)$ is graphene's surface conductivity. For identical resonators $\left(\alpha_{1}=\alpha_{2}\right)$, assuming intraband (Drude) conductivity, we can approximate the ON state radiative thermal conductance (and the corresponding optimal Fermi levels) to emphasize the parameter dependencies as (SI)

$$
\begin{aligned}
& h_{\mathrm{ON}} \approx 116.23\left(\frac{\epsilon_{0} k_{\mathrm{B}}^{5} T^{4}}{2 \pi \hbar^{2} e^{3} v_{\mathrm{F}}^{2}}\right) \frac{1}{A} \frac{\lambda_{S}}{2} \mu \frac{L^{7}}{d^{6}} \frac{\Delta_{1}^{2}}{\zeta_{1}^{3}} \\
& E_{\mathrm{ON}} \approx 71.27\left(\frac{\epsilon_{0} \pi k_{B}^{2} T^{2}}{e^{2}}\right) \frac{L}{\zeta_{1}}
\end{aligned}
$$

which are valid assuming the optical response is dominated by a single (or a set of degenerate) mode(s) associated with $\zeta_{1}, \Delta_{1}$ from (3). Note, $\lambda_{S} \equiv \sum_{j \in \hat{r}} \lambda_{j}$ is the sum of all corresponding numerical pre-factors. For disk resonators of radius $R$, we associate $L \equiv \sqrt{A}=\sqrt{R^{2} \pi}$, and give the relevant oscillator parameters in the SI. Figure 4 shows the normalized maximum thermal conductance $h_{\mathrm{on}} / h_{\mathrm{bb}}$ and the switching ratio $\eta$, for graphene disks of varying size (we assume disks are co-axial, hence $\lambda_{S}=2$ ). We observe that the optimal doping ( $E=E_{1,2}^{\text {on }}$ ) that maximizes the RTC is not particularly sensitive to mobility (Fig. $4 \mathrm{~b}$ ); instead, it is dependent on the resonator size, exhibiting a linear relationship with the disk radius $R$ (in agreement with Eq. 4). The higher optimal doping would seem to imply weaker radiative conductance per unit area between larger disks at temperature $T$ (due to a blue-shifted resonance frequency); nevertheless, the cubic dependence of polarizability on disk size leads to the overall increase of $h_{\text {on }}$ with the disk size, 
as shown in Fig. 4c. We make two remarks. First, while the RTC between identical disks of radius $R$ is proportional to $R^{6}$ for fixed Fermi levels, the optimal conductance (i.e. the ON state) has a stronger $\left(R^{7}\right)$ size dependence (Fig. S4b). Second, Eqs. (3) and (4) are shape-agnostic: they apply to graphene resonators other than disks, allowing for direct comparison between different resonator geometries. For example, using the values from Table S1 (SI), we can readily infer that square, triangular, or elliptical resonators would exhibit stronger on-resonance heat transfer than disks, for the same resonator area. For elliptical resonators, the enhancement arises from the fact that increasing the aspect ratio simultaneously increases the long-axis oscillator strength $\Delta_{1}$ while reducing its eigenfrequency $\zeta_{1}$. For squares and triangles, the argument is more nuanced: both the polarizability and the eigenfrequency are lower relative to disks, but the latter has the stronger effect. Finally, sharp, geometry-dictated resonances lead to orderof-magnitude higher switching ratios relative to those in planar structures (Fig. 4d).

In addition to thermal switching in extended (sheets and multi-layer stacks) and dipolar (e.g. disks) structures, we also analyze a hybrid scenario that combines the two; for example a graphene disk above a single sheet (or a stack) as shown in Fig. 1d. The spectral transfer function of the configuration consisting of a dipolar nanostructure above a planar sheet can be expressed as

$$
\Phi(\omega)=\frac{2 \omega^{2}}{\pi c^{2}} \sum_{i=x, y, z} \operatorname{Im}\left[\alpha_{i}(\omega)\right] \operatorname{Im}\left[\overleftrightarrow{\boldsymbol{G}}\left(\omega, \boldsymbol{r}_{0}\right)\right]_{i i},
$$

where $\overleftrightarrow{\boldsymbol{G}}$ is the dyadic Green tensor of the planar interface (see SI). In the nonretarded limit $(q \gg k)$ relevant to NF RHT, the expression for the spectral transfer function $\Phi(\omega)$ features terms proportional to $\operatorname{Im}(\alpha) \operatorname{Im}\left(r_{p}\right)$, where $\alpha$ is the resonator polarizability and $r_{p}$ is the p-polarization (TM) reflection coefficient for the underlying sheet (SI). Fig. S3 shows the RTC enhancement and the switching ratio, assuming the polarizability of the disk is $\alpha=\sum_{i} \alpha_{i} \hat{n}_{i}=\alpha(1,1,0)^{T}$ where Eq. (3) applies for the scalar $\alpha$. We observe that it is still possible to bring the disk and the sheet into resonance, as indicated by the very large possible switching ratios relative to the sheet/stack configuration of Fig. 2. In contrast to the latter, the inclusion of an additional layer in the stack does not appear to improve either the RHT enhancement or the switching ratio (Fig. S3, dashed). Attainable switching ratios would, in general, depend on the separation between graphene resonators. For the sheet-sheet configuration, Fig. S5 shows the switching ratio as a function of mobility, for different separations. We observe similar trends as before: namely, the switching ratio increases with mobility and that shorter separations are generally favorable due to the enhancement of the ON state conductance as sheets become closer. We note that for resonators in the dipolar limit both the ON and the OFF state energy fluxes scale in the same way with the separation $d$, making the switching ratio insensitive to separation.

Besides the heat transfer enhancement and the switching ratio, another relevant quantity for active modulation is the switching sensitivity. Here, we define the switching sensitivity as $\xi=\mathrm{k}_{\mathrm{B}} T /$ $\min _{i}\left|E_{i}^{\text {on }}-E_{i}^{\text {on/2 }}\right|$, a quantity that is proportional to the minimum change in any single Fermi level $E_{i}$ that is needed to halve the maximum radiative conductance $h_{o n}$. Figure 5 shows the sensitivity $\xi$ for different values of mobility for the discussed configurations. In the disk-disk and the 2-sheet case, the ON state of the system is (due to symmetry) equally sensitive to changes in $E_{1}$ and $E_{2}$. In the 3 -sheet case (Fig 
2., dashed), the most "sensitive" parameter is the doping of the top sheet $\left(E_{1}\right)$; likewise, in the disk-sheet case (Fig. S3, dashed) the doping of the disk $\left(E_{1}\right)$ is the most sensitive. Similar to the switching ratio, the sensitivity of switching increases with increasing graphene mobility, especially for the disk-disk heat transfer characterized by sharp resonances.

Finally, we briefly characterize thermal switching with graphene sheets on substrates. The simplest example comprises a sheet of graphene on a semi-infinite substrate of constant permittivity (e.g. CVD diamond, $\epsilon \sim 5.8$ ). In that case, much of the analysis from Fig. 2 holds, with switching ratios exhibiting similar mobility dependence, with generally lower magnitude due to stronger mode confinement for $\epsilon>1$. A more interesting extension includes the analysis of thermal switching in the presence of IR active substrates (i.e. substrates that themselves support surface electromagnetic modes in the mid-IR). For this case, we focus on $\mathrm{SiO}_{2}, \mathrm{SiC}$, and $\mathrm{SiN}_{\mathrm{x}}$, materials that exhibit surface phononpolaritons. As indicated in Fig. S6, these three materials can be characterized by both sharp and broad resonances as well as by both low and high background permittivities. Figure 6 shows the switching ratio $\eta$ between two graphene sheets on substrates as a function of mobility. The substrates are identical and, as before, we find optimal Fermi levels $E_{i}$ that maximize/minimize the RTC. To emphasize the substrate versus graphene contribution to RTC, we plot the switching ratio for different separation distances $d$. From Fig. 6 we can draw several qualitative conclusions. As expected, for a given substrate, modulation is generally stronger at smaller separations due to enhanced contribution of tightly-confined surface modes in graphene. As a result, at smaller separations (where graphene response is more dominant) higher mobility is still favored. In terms of the most suitable substrate material, silicon carbide appears to provide the largest switching ratio of the analyzed substrates. We attribute this to its narrowest resonant response (as indicated by its permittivity function, Fig. S6) that allows for stronger detuning of the heat transfer in the presence of graphene.

At larger separations, where graphene response is less dominant, the effect of carrier mobility is more nuanced. At a separation of $d=400 \mathrm{~nm}$, we find graphene-on- $\mathrm{SiO}_{2}$ to have the strongest switching ratio. This is attributed to the (comparatively) low $\epsilon_{\mathrm{re}}$ of $\mathrm{SiO}_{2}$, giving rise to less strongly confined surface modes that can more effectively modulate the RTC at such distances. This is the same reason why $\mathrm{SiO}_{2}$ outperforms $\mathrm{SiN}_{\mathrm{x}}$, and even optically inactive CVD diamond, as the substrate material. This implies that, among polaritonic materials, $\mathrm{SiO}_{2}$ may be a suitable substrate for RTC modulation at larger, more experimentally accessible separations.

\section{CONCLUSIONS}

In this work, we proposed and demonstrated a radiative thermal switching scheme with graphene plasmon nanoresonators in several relevant configurations. We showed that optimal combinations of resonator size and carrier concentration give rise to strongly contrasting ON and OFF thermal conductance states, and identified carrier mobility as a critical material parameter. In addition to numerical optimizations, we derived analytical, shape-agnostic approximations that highlight parameter 
dependence for resonant heat transfer and allow for direct comparison between different resonator geometries. Finally, we characterized thermal switching and heat flux modulation of graphene on infrared active substrates. Though the focus of this work is radiative flux modulation via the control of plasmonic resonances in graphene, other reduced-dimensionality materials and other types of polaritons (phononpolariton, exciton-polariton, magnon, etc.) would exhibit similar radiative thermal emission enhancements. In addition to electrostatic gating, other mechanisms, such as an imposed elastic strain, offer another means for polariton resonance modulation. Because of its vanishing density of states at its neutrality point, graphene exhibits exceptional tunability and is particularly suitable for radiative flux modulation. The described active thermal switching may be relevant for applications that include nearfield thermophotovoltaic modulation, and cooling of electronic nano-devices. These results demonstrate the potential of graphene-based plasmonic resonators for active thermal management on the nanoscale.

\section{COMPUTATIONAL METHODS}

Calculations in the present paper were performed by numerical evaluation of Equations (1-5). Unless otherwise specified, optical conductivity of graphene is numerically obtained (for desired values of frequency, Fermi energy, mobility, temperature) by summing the intraband and the interband contributions (see, for example, Ref [47]). For optimizations, the Fermi energy pairs $\left(E_{1,2}^{\text {on }}\right.$ and $\left.E_{1,2}^{\text {off }}\right)$ are computed numerically using a (multi-start) local, derivative-free, optimization algorithms ${ }^{44,45}$, accessed via the NLopt package. ${ }^{43}$

\section{Acknowledgements}

O.I., N.H.T., M.C.S, A.J.M., and H.A.A. were supported as part of the DOE "Light-Material Interactions in Energy Conversion" Energy Frontier Research Center funded by the US Department of Energy, Office of Science, Office of Basic Energy Sciences under Award Number DE-SC0001293. O.I., M.C.S., and H.A.A. acknowledge support from the Northrop Grumman Corporation through NG Next. M.C.S. acknowledges fellowship support from the Resnick Sustainability Institute. O.D.M. was supported by the Air Force Office of Scientific Research under Award Number FA9550-17-1-0093. T.C. was supported by the Danish Council for Independent Research (Grant DFFC6108-00667). M.S. was supported as part of the Army Research Office through the Institute for Soldier Nanotechnologies under contract no. W911NF-13-D-0001 (photon management for developing nuclear-TPV and fuel-TPV mmscale-systems). M.S. was also supported as part of the S3TEC, an Energy Frontier Research Center funded by the US Department of Energy under grant no. DE-SC0001299 (for fundamental photon transport related to solar TPVs and solar-TEs).

\section{Supporting Information}

The Supporting Information is available free of charge on the ACS Publications website at DOI: ------Analysis beyond the local response approximation (LRA) conductivity; expressions for the radiative heat transfer involving dipolar structures; polarizability model for graphene 
resonators; first-order approximations to the radiative thermal conductance; separation distance; effect of substrate (PDF).

\section{References}

(1) Hargreaves, C. M. Anomalous Radiative Transfer between Closely-Spaced Bodies. Phys. Lett. A 1969, 30, 491-492.

(2) Polder, D.; Van Hove, M. Theory of Radiative Heat Transfer between Closely Spaced Bodies. Phys. Rev. B 1971, 4, 3303-3314.

(3) Narayanaswamy, A.; Shen, S.; Chen, G. Near-Field Radiative Heat Transfer between a Sphere and a Substrate. Phys. Rev. B 2008, 78, 115303.

(4) Shen, S.; Narayanaswamy, A.; Chen, G. Surface Phonon Polaritons Mediated Energy Transfer between Nanoscale Gaps. Nano Lett. 2009, 9, 2909-2913.

(5) Rousseau, E.; Siria, A.; Jourdan, G.; Volz, S.; Comin, F.; Chevrier, J.; Greffet, J.-J. Radiative Heat Transfer at the Nanoscale. Nat. Photonics 2009, 3, 514-517.

(6) Ottens, R. S.; Quetschke, V.; Wise, S.; Alemi, A. A.; Lundock, R.; Mueller, G.; Reitze, D. H.; Tanner, D. B.; Whiting, B. F. Near-Field Radiative Heat Transfer between Macroscopic Planar Surfaces. Phys. Rev. Lett. 2011, 107, 14301.

(7) Guha, B.; Otey, C.; Poitras, C. B.; Fan, S.; Lipson, M. Near-Field Radiative Cooling of Nanostructures. Nano Lett. 2012, 12, 4546-4550.

(8) van Zwol, P. J.; Thiele, S.; Berger, C.; de Heer, W. A.; Chevrier, J. Nanoscale Radiative Heat Flow due to Surface Plasmons in Graphene and Doped Silicon. Phys. Rev. Lett. 2012, 109, 264301.

(9) St-Gelais, R.; Guha, B.; Zhu, L.; Fan, S.; Lipson, M. Demonstration of Strong Near-Field Radiative Heat Transfer between Integrated Nanostructures. Nano Lett. 2014, 14, 6971-6975.

(10) Song, B.; Ganjeh, Y.; Sadat, S.; Thompson, D.; Fiorino, A.; Fernández-Hurtado, V.; Feist, J.; Garcia-Vidal, F. J.; Cuevas, J. C.; Reddy, P.; Meyhofer, E. Enhancement of Near-Field Radiative Heat Transfer Using Polar Dielectric Thin Films. Nat. Nanotechnol. 2015, 10, 253-258.

(11) Kim, K.; Song, B.; Fernández-Hurtado, V.; Lee, W.; Jeong, W.; Cui, L.; Thompson, D.; Feist, J.; Reid, M. T. H.; Garcia-Vidal, F. J.; Cuevas, J. C; Meyhofer, E.; Reddy, P. Radiative Heat Transfer in the Extreme Near Field. Nature 2015, 528, 387-391.

(12) Ito, K.; Miura, A.; Iizuka, H.; Toshiyoshi, H. Parallel-Plate Submicron Gap Formed by Micromachined Low-Density Pillars for near-Field Radiative Heat Transfer. Appl. Phys. Lett. 2015, 106, 83504.

(13) St-Gelais, R.; Zhu, L.; Fan, S.; Lipson, M. Near-Field Radiative Heat Transfer between Parallel Structures in the Deep Subwavelength Regime. Nat. Nanotechnol. 2016, 11, 515-519.

(14) Bernardi, M. P.; Milovich, D.; Francoeur, M. Radiative Heat Transfer Exceeding the Blackbody Limit between Macroscale Planar Surfaces Separated by a Nanosize Vacuum Gap. Nat. Commun. 2016, 7, 12900.

(15) Watjen, J. I.; Zhao, B.; Zhang, Z. M. Near-Field Radiative Heat Transfer between Doped-Si Parallel Plates Separated by a Spacing down to $200 \mathrm{~nm}$. Appl. Phys. Lett. 2016, 109, 203112.

(16) Kloppstech, K.; Könne, N.; Biehs, S.-A.; Rodriguez, A. W.; Worbes, L.; Hellmann, D.; Kittel, A. Giant Heat Transfer in the Crossover Regime between Conduction and Radiation. Nat. Commun. 2017, 8, 14475.

(17) Cui, L.; Jeong, W.; Fernández-Hurtado, V.; Feist, J.; Garcia-Vidal, F. J.; Cuevas, J. C.; Meyhofer, E.; Reddy, P. Study of Radiative Heat Transfer in Ångström- and Nanometre-Sized Gaps. Nat. Commun. 2017, 8, 14479. 
(18) Liu, X.; Zhang, Z. Near-Field Thermal Radiation between Metasurfaces. ACS Photonics 2015, 2, $1320-1326$.

(19) Zhu, L.; Fan, S. Persistent Directional Current at Equilibrium in Nonreciprocal Many-Body Near Field Electromagnetic Heat Transfer. Phys. Rev. Lett. 2016, 117, 134303.

(20) Khandekar, C.; Jin, W.; Miller, O. D.; Pick, A.; Rodriguez, A. W. Giant Frequency-Selective Near-Field Energy Transfer in Active-Passive Structures. Phys. Rev. B 2016, 94, 115402.

(21) Principi, A.; Lundeberg, M. B.; Hesp, N. C. H.; Tielrooij, K.-J.; Koppens, F. H. L.; Polini, M. Super-Planckian Electron Cooling in a van Der Waals Stack. Phys. Rev. Lett. 2017, 118, 126804.

(22) Chen, K.; Santhanam, P.; Fan, S. Near-Field Enhanced Negative Luminescent Refrigeration. Phys. Rev. Appl. 2016, 6, 024014.

(23) Ding, D.; Kim, T.; Minnich, A. J. Active Thermal Extraction of Near-Field Thermal Radiation. Phys. Rev. B 2016, 93, 081402.

(24) Otey, C. R.; Lau, W. T.; Fan, S. Thermal Rectification through Vacuum. Phys. Rev. Lett. 2010, 104, 154301.

(25) Iizuka, H.; Fan, S. Rectification of Evanescent Heat Transfer between Dielectric-Coated and Uncoated Silicon Carbide Plates. J. Appl. Phys. 2012, 112, 24304.

(26) Zhu, L.; Otey, C. R.; Fan, S. Ultrahigh-Contrast and Large-Bandwidth Thermal Rectification in Near-Field Electromagnetic Thermal Transfer between Nanoparticles. Phys. Rev. B 2013, 88, 184301.

(27) Ben-Abdallah, P.; Biehs, S.-A. Near-Field Thermal Transistor. Phys. Rev. Lett. 2014, 112, 044301.

(28) Ben-Abdallah, P.; Joulain, K. Fundamental Limits for Noncontact Transfers between Two Bodies. Phys. Rev. B 2010, 82, 121419.

(29) Miller, O. D.; Johnson, S. G.; Rodriguez, A. W. Shape-Independent Limits to Near-Field Radiative Heat Transfer. Phys. Rev. Lett. 2015, 115, 204302.

(30) Miller, O. D.; Ilic, O.; Christensen, T.; Reid, M. T. H.; Atwater, H. A.; Joannopoulos, J. D.; Soljačić, M.; Johnson, S. G. Limits to the Optical Response of Graphene and Two-Dimensional Materials. Nano Lett. 2017, 17, 5408-5415.

(31) Volokitin, A. I.; Persson, B. N. J. Near-Field Radiative Heat Transfer between Closely Spaced Graphene and Amorphous $\mathrm{SiO}_{2}$. Phys. Rev. B 2011, 83, 241407.

(32) Ilic, O.; Jablan, M.; Joannopoulos, J. D.; Celanovic, I.; Soljačić, M. Overcoming the Black Body Limit in Plasmonic and Graphene near-Field Thermophotovoltaic Systems. Opt. Express 2012, 20, A366.

(33) Ilic, O.; Jablan, M.; Joannopoulos, J. D.; Celanovic, I.; Buljan, H.; Soljačić, M. Near-Field Thermal Radiation Transfer Controlled by Plasmons in Graphene. Phys. Rev. B 2012, 85, 155422.

(34) Svetovoy, V. B.; van Zwol, P. J.; Chevrier, J. Plasmon Enhanced near-Field Radiative Heat Transfer for Graphene Covered Dielectrics. Phys. Rev. B 2012, 85, 155418.

(35) Messina, R.; Hugonin, J.-P.; Greffet, J.-J.; Marquier, F.; De Wilde, Y.; Belarouci, A.; Frechette, L.; Cordier, Y.; Ben-Abdallah, P. Tuning the Electromagnetic Local Density of States in Graphene-Covered Systems via Strong Coupling with Graphene Plasmons. Phys. Rev. B 2013, 87, 085421.

(36) Liu, X. L.; Zhang, Z. M. Graphene-Assisted Near-Field Radiative Heat Transfer between Corrugated Polar Materials. Appl. Phys. Lett. 2014, 104, 251911.

(37) Ben-Abdallah, P.; Belarouci, A.; Frechette, L.; Biehs, S.-A. Heat Flux Splitter for near-Field Thermal Radiation. Appl. Phys. Lett. 2015, 107, 53109.

(38) Yang, Y.; Wang, L. Electrically-Controlled Near-Field Radiative Thermal Modulator Made of Graphene-Coated Silicon Carbide Plates. J. Quant. Spectrosc. Radiat. Transf. 2017, 197, 68-75.

(39) Yu, R.; Manjavacas, A.; de Abajo, F. J. Ultrafast Radiative Heat Transfer. Nat. Commun. 2017, 8, 2.

(40) Ramirez, F. V; Shen, S.; McGaughey, A. J. H. Near-Field Radiative Heat Transfer in Graphene Plasmonic Nanodisk Dimers. Phys. Rev. B 2017, 96, 165427.

(41) Rodriguez, A. W.; Ilic, O.; Bermel, P.; Celanovic, I.; Joannopoulos, J. D.; Soljačić, M.; Johnson, 
S. G. Frequency-Selective Near-Field Radiative Heat Transfer between Photonic Crystal Slabs: A Computational Approach for Arbitrary Geometries and Materials. Phys. Rev. Lett. 2011, 107, 114302.

(42) Jablan, M.; Buljan, H.; Soljačić, M. Plasmonics in Graphene at Infrared Frequencies. Phys. Rev. B 2009, 80, 245435.

(43) Johnson, S. G. The NLopt nonlinear-optimization package http://ab-initio.mit.edu/nlopt.

(44) Rinnooy Kan, A. H. G.; Timmer, G. T. Stochastic Global Optimization Methods. Math. Program. 1987, 39, 27-78.

(45) Powell, M. J. D. The BOBYQA Algorithm for Bound Constrained Optimization without Derivatives; Cambridge NA Report NA2009/06, University of Cambridge, Cambridge. 2009, 2646.

(46) Novotny, L.; Hecht, B. Principles of Nano-Optics; Cambridge University Press, 2006.

(47) Christensen, T. From Classical to Quantum Plasmonics in Three and Two Dimensions, PhD Thesis, Technical University of Denmark, 2015. 
(a) Two sheets

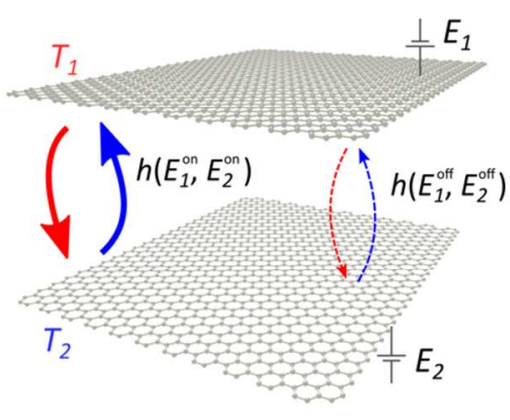

(c) Di/multi polar resonators

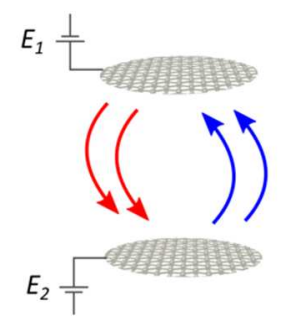

(b) Parallel stacks

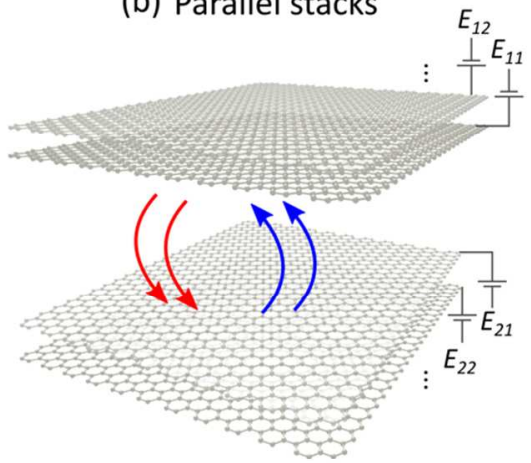

(d) Hybrid structures

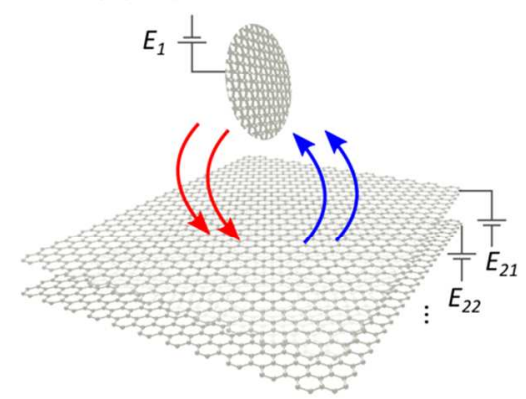

Fig 1. Operating principle for a radiative thermal switch using graphene plasmon nanoresonators in structures comprising $(a, b)$ parallel (multi)layers, (c) di/multipolar resonators, and (d) hybrid configurations. External control of the relevant Fermi levels $E_{i}=\left(E_{1}, \ldots\right)$ modulates the nearfield heat transfer between the "OFF" state at minimal radiative thermal conductance $h_{\text {off }}=$ $h\left(E_{1}^{\text {off }}, \ldots\right)$, and the "ON" state at maximal conductance $h_{\mathrm{on}}=h\left(E_{1}^{\mathrm{on}}, \ldots\right)$. 

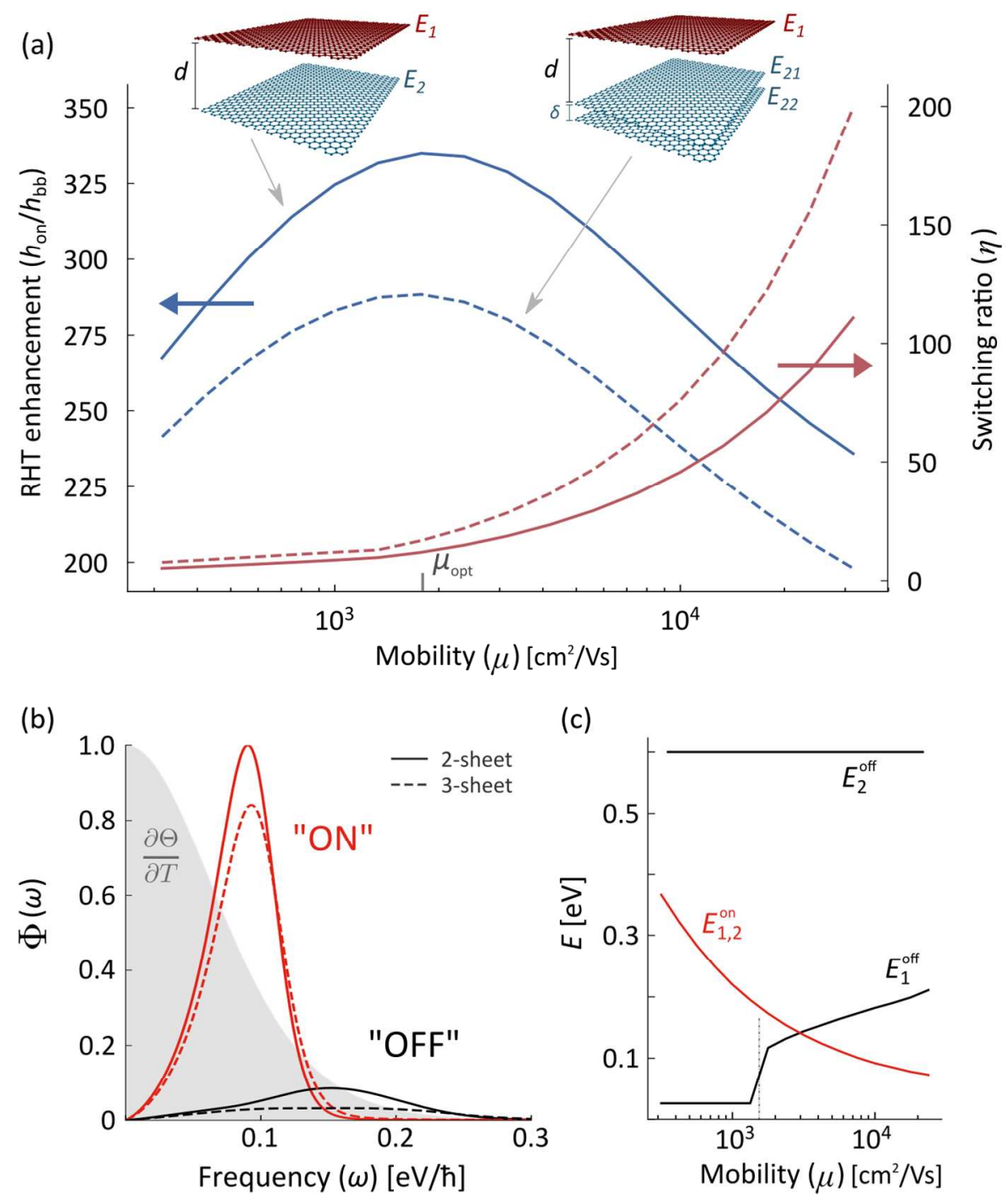

Fig 2. Thermal switching in parallel graphene stacks. (a) RHT enhancement $h_{\mathrm{on}} / h_{\mathrm{bb}}$ (left) and the switching ratio $\eta=h_{\text {on }} / h_{\text {off }}$ (right) for a 2-sheet (solid) and a 3-sheet (dashed) configuration, as a function of mobility $\mu_{1,2}=\mu$. (b) Spectral heat flux indicating the "ON" and the "OFF" states for mobility $\mu=\mu_{\mathrm{opt}}$ from (a). Shaded region shows Planck's prefactor from (1). (c) Corresponding Fermi levels for the "ON" $\left(E_{1,2}^{\text {on }}\right)$ and the "OFF" $\left(E_{1,2}^{\text {off }}\right)$ states in the 2sheet case. Here, $T=300 \mathrm{~K}, d=100 \mathrm{~nm}, \delta=10 \mathrm{~nm}$. 

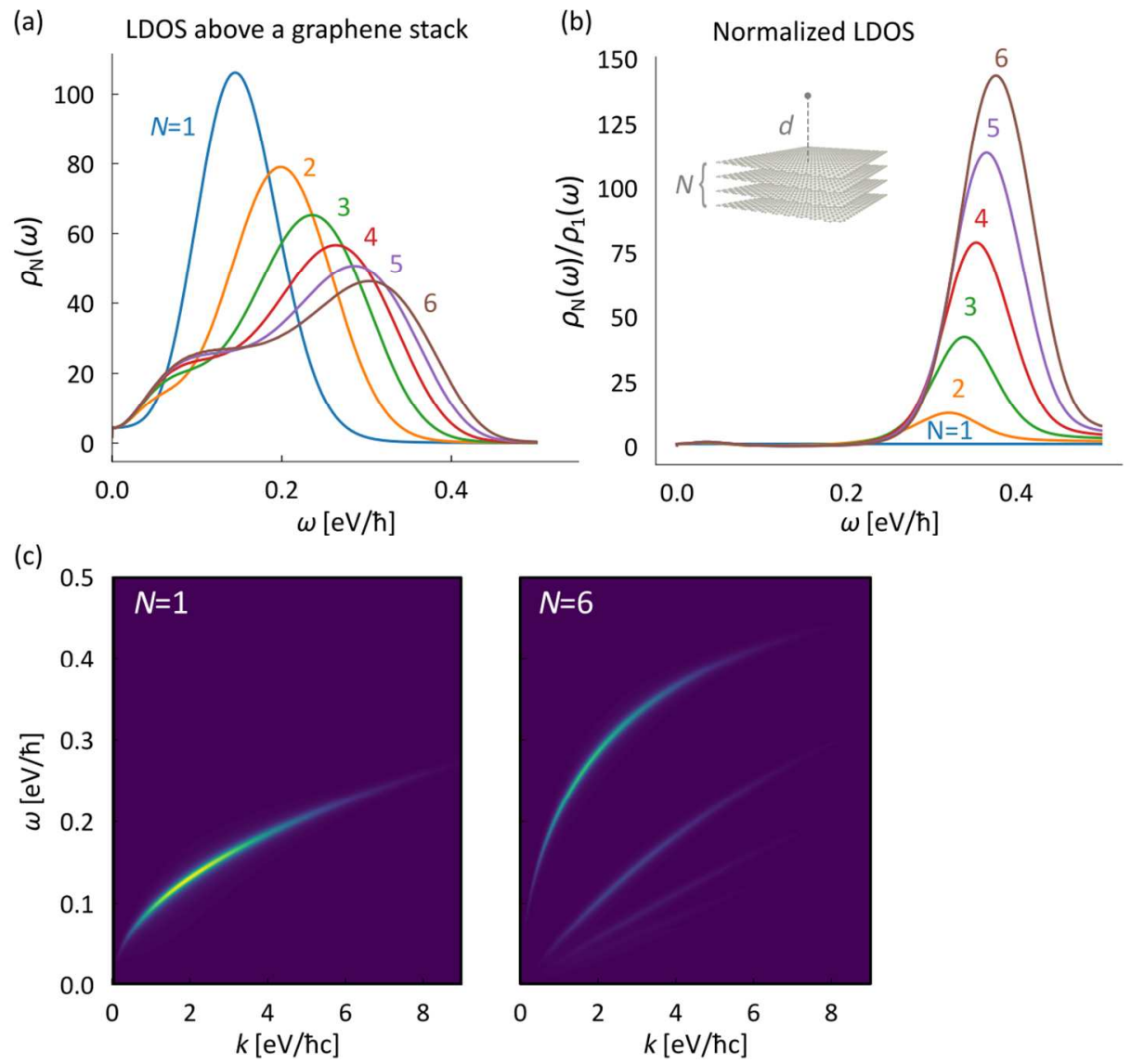

Fig 3. (a) Orientation-averaged local density of states (LDOS) $\rho$ at a height $d$ above a graphene stack of $N$ identical sheets $\left(E_{i}=0.6 \mathrm{eV}\right)$, separated by $\delta$ (see inset in (b)). (b) LDOS for $N>1$ normalized to that of $N=1$. (c) Decomposed $(k, \omega)$ LDOS for $N=1$ and $N=6$. Here, $d=100 \mathrm{~nm}, \delta=10 \mathrm{~nm}$. 

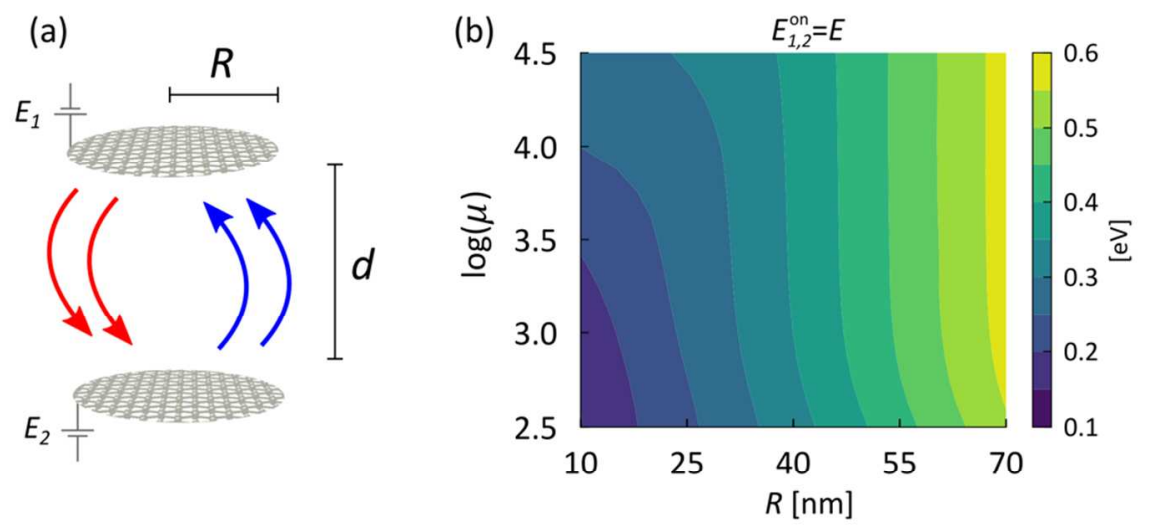

(c)

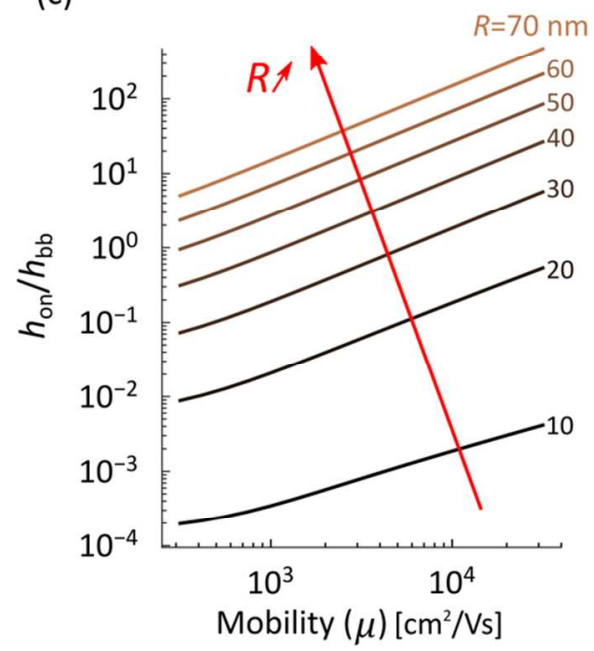

(d)

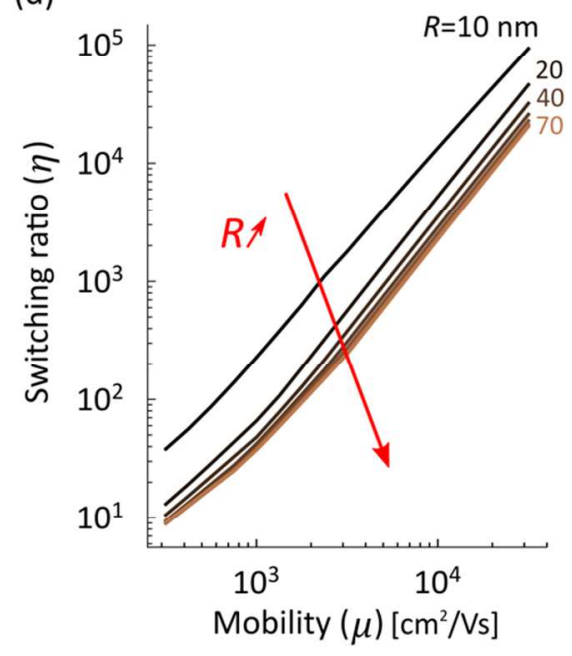

Fig 4. Radiative thermal switching between parallel graphene disks (a). Optimal carrier concentration levels $E_{1,2}^{\text {on }}=E$ for the "ON" state (b), RHT enhancement (c), and the switching ratio (d), as a function of mobility $(\mu)$ and disk radius $(R)$. Here, $T=300 \mathrm{~K}, d=200 \mathrm{~nm}$. 


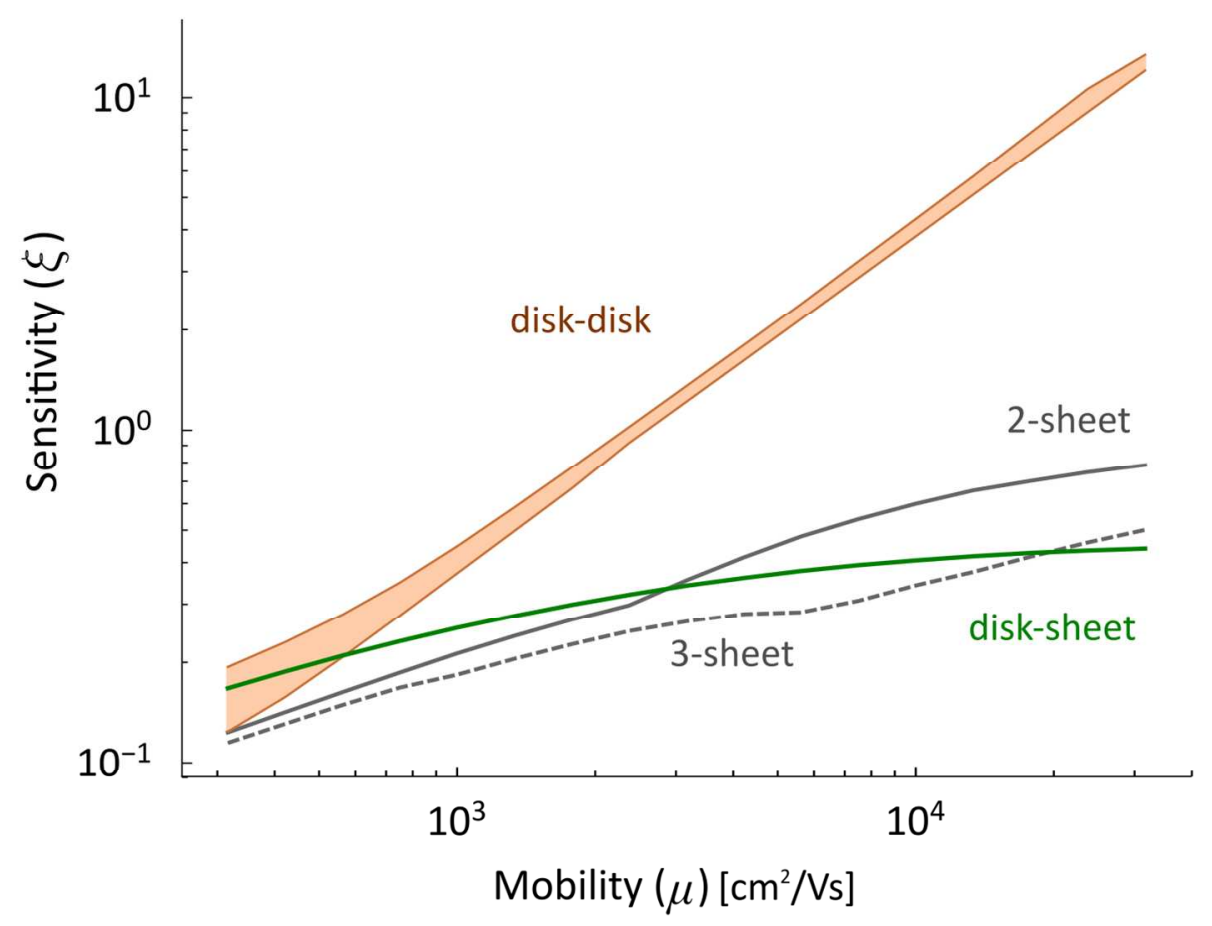

Fig 5. Sensitivity of thermal switching defined as $\xi=\mathrm{k}_{\mathrm{B}} T / \min _{i}\left|E_{i}^{\mathrm{on}}-E_{i}^{\mathrm{on} / 2}\right|$ (i.e. inversely proportional to the smallest change in $E_{i}$ needed to halve the "ON" state thermal conductance), for resonator configurations from Fig. 1. For parallel disks (Fig. 4), a range from $R=10 \mathrm{~nm}$ (most sensitive) to $R=70 \mathrm{~nm}$ (least sensitive) is shown. 


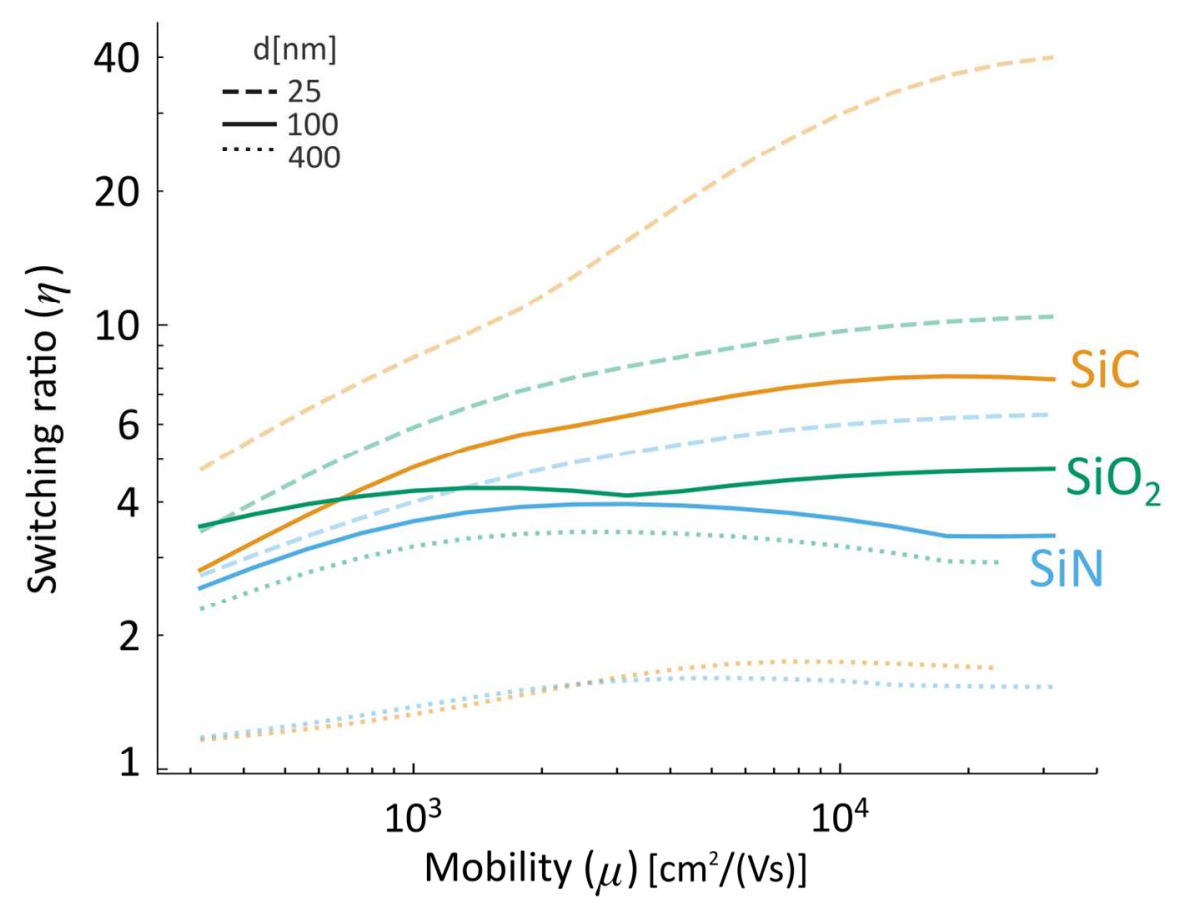

Fig 6. Thermal switching between parallel graphene sheets (Fig. 1a), now on identical substrates that support surface phonon-polaritons $\left(\mathrm{SiC}, \mathrm{SiN}\right.$, or $\left.\mathrm{SiO}_{2}\right)$. Different line styles indicate the switching ratio $\eta$ for separation distance $d$ of $25 \mathrm{~nm}$ (dashed), 100 $\mathrm{nm}$ (solid), and $400 \mathrm{~nm}$ (dotted) $(T=300 \mathrm{~K})$. As before, for each value of mobility, the respective Fermi levels for the $\mathrm{ON}\left(E_{1,2}^{\text {on }}\right)$ and the $\mathrm{OFF}\left(E_{1,2}^{\text {off }}\right)$ state are determined. 